\title{
Efficacy through BibNAbs
}

An increasing number of broadly neutralizing antibodies (bNAbs) against HIV-1 are being identified, and efforts are underway to optimize these for passive immunization regimens. As yet, their capacity to neutralize different HIV-1 strains is limited. Now, reporting in PNAS, Ho and colleagues present bispecific bNAbs (BibNAbs) with exceptional potency against all HIV-1 strains tested.

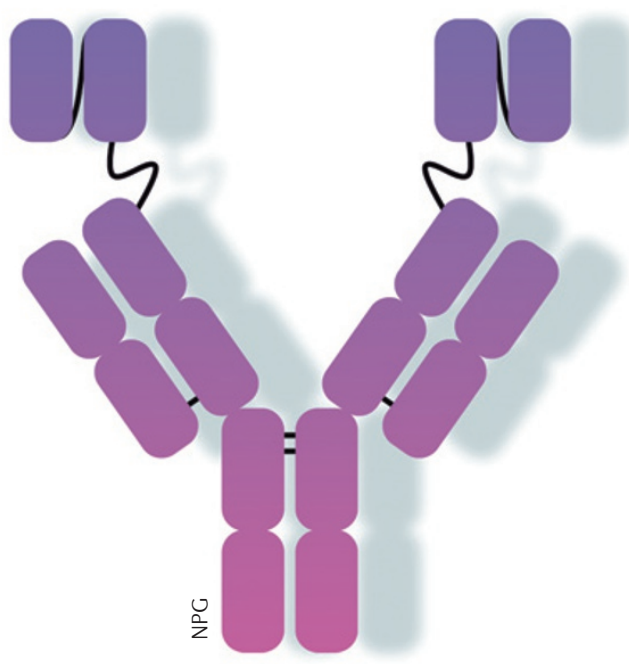

The BibNAbs were designed using the humanized monoclonal antibody ibalizumab as a scaffold. Ibalizumab (which has a proven safety record in clinical trials) targets CD4, which is the receptor that serves as an entry point for HIV-1 on human T cells. Ibalizumab inhibits HIV-1 entry via a non-competitive mechanism; it binds the CD4 domain 2 on a face opposite to the site of viral gp120 binding, and blocks viral entry after virus attachment.

The authors fused a linker to the amino terminus of the heavy chain variable region of ibalizumab, and used this linker to engraft single-chain variable fragments (scFvs) of different bNAbs specific for the gp120 subunit of the envelope protein (ENV) of HIV-1. This effectively created antibodies with four antigen-binding regions that are specific for two different targets.

Two such constructs had remarkable anti-HIV-1 profiles. In vitro experiments showed that they inhibited a panel of 118 diverse HIV-1 strains at picomolar concentrations and even neutralized viruses that are resistant to both parent antibodies. The BibNAbs blocked viral entry more efficiently than a combination of five highly potent bNAbs or a mixture of the parent bNAbs.
This indicates a synergistic activity of the antibody-binding regions of these constructs.

Both the geometry of the antibody construct (that is, whether the scFV is engrafted to the amino or carboxyl terminus of the scaffold) and the length of the linker influenced neutralization activity. Further investigation revealed that the increase in potency of the BibNAb compared to the parent antibodies is due to the ability of the BibNab to anchor the active anti-ENV moiety to the point of viral entry on the target cell, thereby increasing its local concentration at the precise point where it is needed.

The authors suggest that these BibNAbs possess the requisite antiviral breadth and potency for passive immunization. Importantly, and in contrast to current pre-exposure prophylaxis regimens with antiretrovirals that need daily administration, the long half-life of antibodies means that BibNAbs may only require monthly or bimonthly injection a factor that could greatly increase patient compliance to a prophylactic regimen.

Alexandra Flemming

ORIGINAL RESEARCH PAPER Pace, C. S. et al. Bispecific antibodies directed to $\mathrm{CD} 4$ domain 2 and HIV envelope exhibit exceptional breadth and picomolar potency against HIV-1. Proc. Natl. Acad. Sci. USA 2013 (dx.doi:10.1073/pnas.1304985110) 